\title{
A generic model for selective adaptation in networks of heterogeneous populations

\author{
Avner Wallach ${ }^{1}$, Danny Eytan ${ }^{1}$, Shimon Marom² and Ron Meir*1
}

\author{
Address: ${ }^{1}$ Department of Electrical Engineering, Technion, Haifa 32000, Israel and ${ }^{2}$ Faculty of Medicine, Technion, Haifa 31096, Israel \\ Email: Ron Meir* - rmeir@ee.technion.ac.il \\ * Corresponding author
}

from Sixteenth Annual Computational Neuroscience Meeting: CNS*2007

Toronto, Canada. 7-12 July 2007

Published: 6 July 2007

BMC Neuroscience 2007, 8(Suppl 2):PI83 doi:I0.I I86/I47I-2202-8-S2-PI83

(c) 2007 Wallach et al; licensee BioMed Central Ltd.

Adaptation is a biologically ubiquitous process whereby features of the system's responsiveness change as a result of persistent input. Most often, the kinetics of the change are monotonic and depend upon the input frequency. Adaptation in neural systems is inherently selective to the input characteristics; not only between sensory modalities, but even within a given modality, the system is capable of reducing its sensitivity to frequent input while preserving (or even enhancing) its sensitivity to the rare (e.g. [1-4]). In-vivo analyses suggest that a within-modality selective adaptation does not require concrete, precise point-to-point wiring (which would be a trivial yet nonphysiological realization) [5]. Indeed, theoretical considerations indicate that, for the case of a single neuron, selective adaptation can be explained in terms of synaptic population dynamics (e.g. [6]). In-vitro analyses in networks of cortical neurons show that, beyond temporal dynamics, differences between topologies of excitatory and inhibitory sub-networks account for the full range of selective adaptation phenomena, including increased sensitivity to the rare [7]. Formal descriptions of selective adaptation are hindered by the problem of representing these different topologies in an analytically useful manner. In this study we offer a formalism that expresses topologies of connectivity in terms of temporal input gain modulation. Using this technique, we are able to formulate a generic analytic model for selective adaptation, which reconstructs all the major experimentally observed phenomena, offers predictions for further experimental analyses, and caters for a rigorous characterization of adaptation in general, and selective adaptation in particular.

\section{References}

I. Tiitinen H, May P, Reinikainen K, Naatanen R: Attentive novelty detection in humans is governed by pre-attentive sensory memory. Nature 1994, 372:90-92.

2. Dragoi V, Sharma J, Sur M: Adaptation-induced plasticity of orientation tuning in adult visual cortex. Neuron 2000, 28:287-298.

3. Naatanen R, Tervaniemi M, Sussman E, Paavilainen P, Winkler I: "Primitive intelligence" in the auditory cortex. Trends Neurosci 200I, 24:283-288.

4. Opitz B, Rinne T, Mecklinger A, von Cramon DY, Schroger E: Differential contribution of frontal and temporal cortices to auditory change detection: fMRI and ERP results. Neuroimage 2002, I5:167-174.

5. Ulanovsky N, Las L, Nelken I: Processing of low-probability sounds by cortical neurons. Nat Neurosci 2003, 6:391-398.

6. Abbott LF, Varela JA, Sen K, Nelson SB: Synaptic depression and cortical gain control. Science 1997, 275:220-224.

7. Eytan D, Brenner N, Marom S: Selective adaptation in networks of cortical neurons. J Neurosci 2003, 23:9349-9356. 